$$
\begin{gathered}
\text { DOE } / E W / 12823-75 \\
\text { CDR95-073 } \\
\text { PEGGERLED } \\
\text { APRO4:4996 } \\
\text { OSATI }
\end{gathered}
$$

\title{
Remote Metrology System (RMS) Design Concept
}

\author{
Contract No.: 15X-ST362V
}

October 19, 1995

\section{Submitted by:}

Coleman Research Corporation Sensor and Intelligence Systems Group 6551 Loisdale Court, Suite 800

Springfield, Virginia 22150

\section{Submitted to:}

Martin Marietta Energy Systems, Inc. Attn: Mr. R. A. Gordon P.O. Box 2002

Oak Ridge, Tennessee 37831-6501 


\section{DISCLAIMER}

This report was prepared as an account of work sponsored by an agency of the United States Government. Neither the United States Government nor any agency thereof, nor any of their employees, make any warranty, express or implied, or assumes any legal liability or responsibility for the accuracy, completeness, or usefulness of any information, apparatus, product, or process disclosed, or represents that its use would not infringe privately owned rights. Reference herein to any specific commercial product, process, or service by trade name, trademark, manufacturer, or otherwise does not necessarily constitute or imply its endorsement, recommendation, or favoring by the United States Government or any agency thereof. The views and opinions of authors expressed herein do not necessarily state or reflect those of the United States Government or any agency thereof. 


\section{DISCLAIMER}

Portions of this document may be illegible in electronic image products. Images are produced from the best available original document. 


\section{Table of Contents}

Section Title

Page

1.0

INTRODUCTION 1

System Configuration $. \ldots \ldots \ldots \ldots \ldots \ldots \ldots \ldots \ldots \ldots \ldots \ldots \ldots \ldots, \ldots$

RMS Concept of Operation.$\ldots \ldots \ldots \ldots \ldots \ldots \ldots \ldots \ldots \ldots \ldots \ldots \ldots$

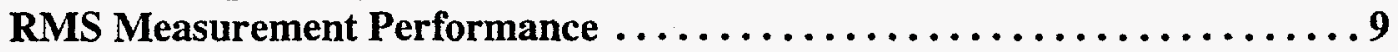

Range Measurement $\ldots \ldots \ldots \ldots \ldots \ldots \ldots \ldots \ldots \ldots \ldots \ldots . \ldots \ldots$

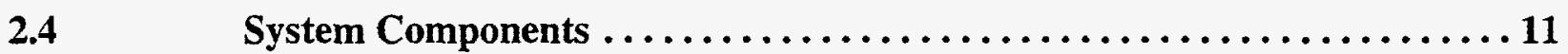

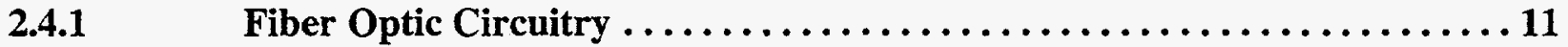

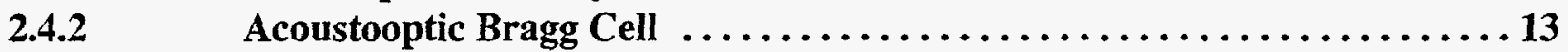

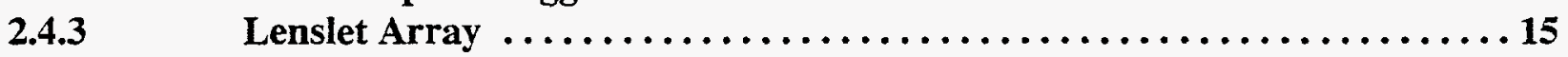

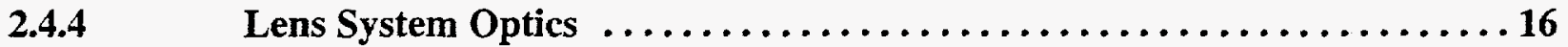

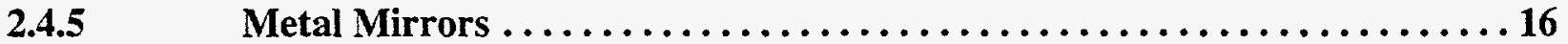

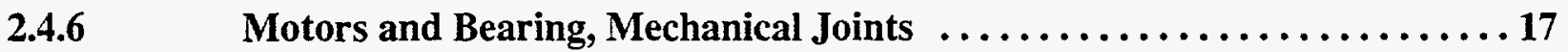

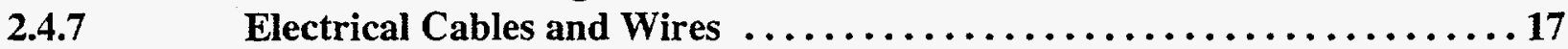

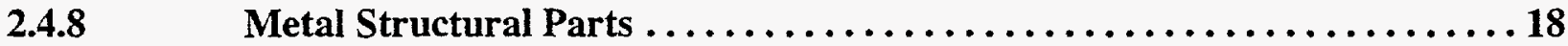

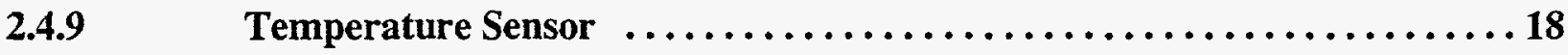

2.5 Alternative RMS Design Approach (Switched Fiber Optic Scanner) . . . . 18

3.0 CONCLUSION AND RECOMMENDATIONS $\ldots \ldots \ldots \ldots \ldots \ldots \ldots$

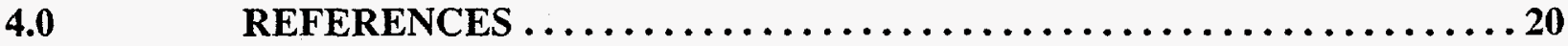

APPENDIX A Performance Estimate for the ITER RMS, a Fiber Optic FMCW

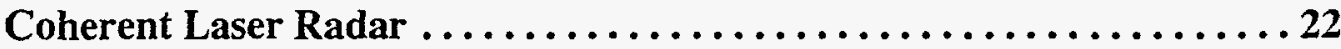




\subsection{INTRODUCTION}

A 3D remote metrology system (RMS) is needed to map the interior plasma-facing components of the International Thermonuclear Experimental Reactor (ITER). The performance and survival of these components within the reactor vessel are strongly dependent on their precise alignment and positioning with respect to the plasma edge. Without proper positioning and alignment, plasma-facing surfaces will erode rapidly.

The metrology system will be used to verify geometry of the as-built configuration and of the configuration after operational shutdown. As a result the system will have to withstand a radiation environment of $3 \times 10^{6} \mathrm{rad} / \mathrm{hr} \gamma$ radiation, $200^{\circ} \mathrm{C}$ interior temperatures, and a high (10 ${ }^{-8}$ Torr) vacuum which is maintained between reactor operations.

A RMS design involving Coleman Research Corporation (CRC) fiber optic coherent laser radar (CLR) technology is examined in this study. The fiber optic CLR approach was selected because its high precision should be able to meet the ITER $0.1 \mathrm{~mm}$ accuracy requirement and because the CLR's fiber optic implementation allows a 3D scanner to operate remotely from the RMS system's vulnerable components.

This design study has largely verified that a fiber optic CLR based RMS can survive the ITER environment and map the ITER interior at the required accuracy at a one measurement/ $\mathrm{cm}^{2}$ density with a total measurement time of less than one hour from each of six or more vertically deployed measurement probes (Figure 1-1). The design approach employs a sealed and pressurized measurement probe which is attached with an umbilical spiral bellows conduit. This conduit bears fiber optic and electronic links plus a stream of air to lower the temperature in the interior of the probe.

Lowering the probe temperature is desirable because probe electromechanical components which could survive the radiation environment often were not rated for the $200^{\circ} \mathrm{C}$ temperature.

The tip of the probe whose outer shell has a flexible bellows joint can swivel in two degrees of freedom to allow mapping operations at each probe deployment level.

This design study has concluded that the most successful scanner design will involve a hybrid AO beam deflector and mechanical scanner. This provides the best combination of high mapping speed and low signal loss (high measurement accuracy) for ITER requirements. A single mode fiber optic circuit approach will be used to avoid the higher radiation sensitivity of polarization maintaining fibers. Further radiation tests and radiation - temperature tests are required for $\mathrm{TeO}_{2} \mathrm{AO}$ Bragg cells and for rad hardened PM fibers. Tests of the probe cooling approach are needed to define the temperature requirement for probe interior components. 


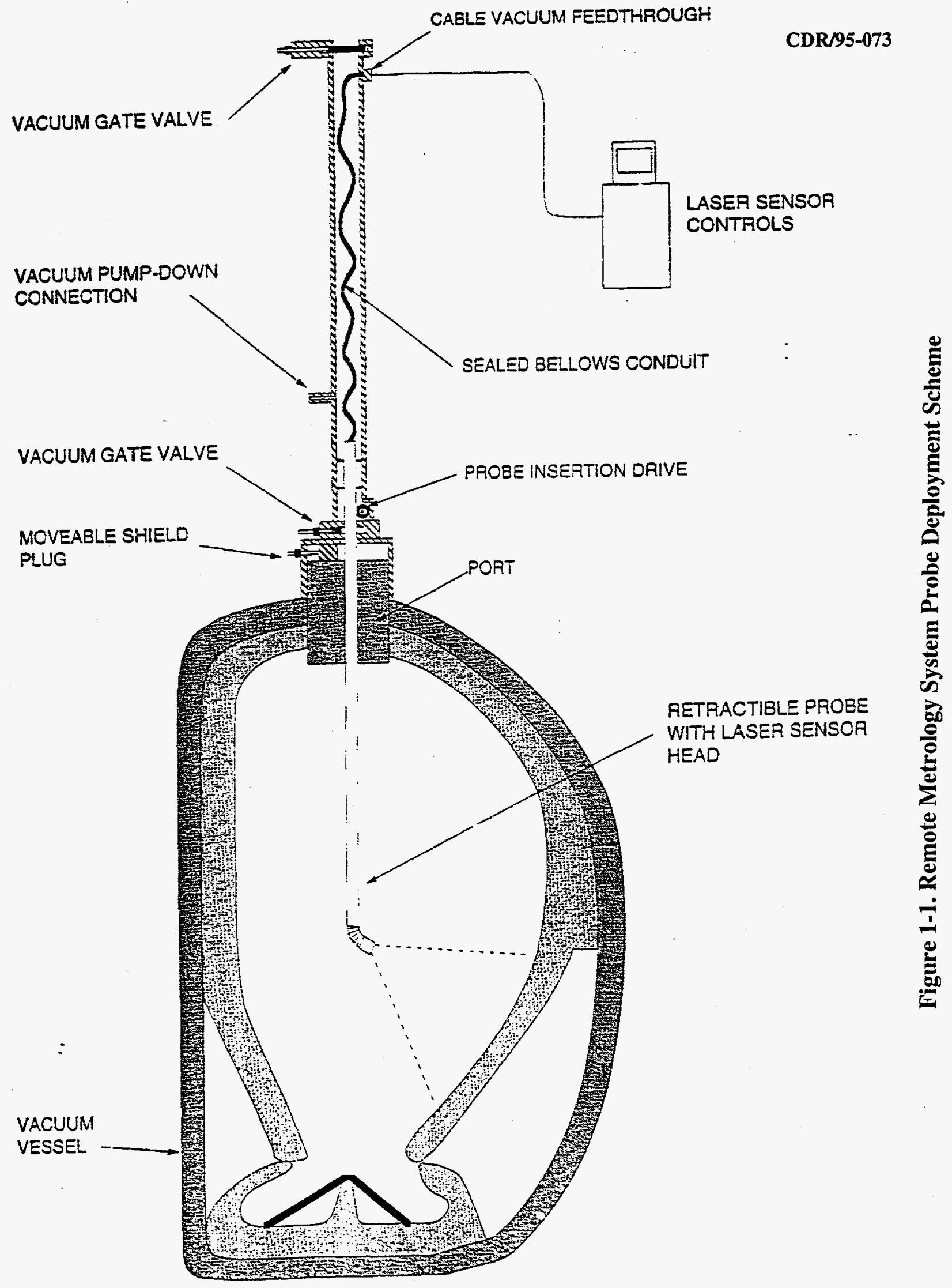


In the event that Bragg cell radiation tests provide positive results, at least $10^{8}$ rad RMS system life without component replacement, or 33 hours of $3 \times 10^{6} \mathrm{rads} /$ hour is expected. 


\subsection{THE RMS SYSTEM DESIGN}

\subsection{System Configuration}

\subsubsection{Coherent Laser Radar as used in RMS}

The RMS will employ the fiber optic FMCW coherent laser radar (CLR) technology developed by CRC to perform its range measurements. This technology and the basis of its performance estimates is discussed in detail in Appendix A.

The block diagram of the CLR currently used in CRC's 3D precision mapping systems is shown in Figure 2-1. The lightwave signal from the laser source sweeps linearly in frequency with time. Lightwave signals which have traveled to the target or through the local oscillator fiber optic loop will have a delay which results in a constant beat frequency when the two signals are mixed at the detector. This beat frequency is proportional the path length difference between the target path and the local oscillator path.

The FMCW coherent laser radar has a number of advantages compared with conventional AM laser radars:

- Wide bandwidth (approaching $60 \mathrm{GHz}$ for RMS) yields high precision for a short measurement dwell

- Radiation resistant fiber optic implementation

- Digital receiver is stable, no draft

- $\quad$ FM detection is not confused by surface brightness features

\subsubsection{Remote Metrology System (RMS) Scanner Probe}

The ITER Remote Metrology System (RMS) Probe design concept is shown in Figure 1-1. The RMS probe is lowered into the ITER interior chamber at a succession of vertical levels from which takes place the mapping of the interior surfaces best viewed from each level. The outer surface of the probe is a vacuum jacket which protects the purity of the vacuum in the ITER chamber and, at the same time, allows an atmosphere to exist inside the probe to support cooling and to prevent adhesion of metal parts in the mechanical joints. A metal bellows in the vacuum sleeve allows a mechanical arm in the interior of the sleeve to rotate in the elevation direction (see Figure 2-2). A flat glass window provides for undistorted viewing by the interior scanner assembly. The mechanical arm may rotate freely inside the sleeve which rides on bearings below the bellows and below the elevation joint of the mechanical arm. The azimuth joint of the mechanical arm is above the bellows. In practice, it may be necessary to adjust the azimuth joint only when the elevation joint 


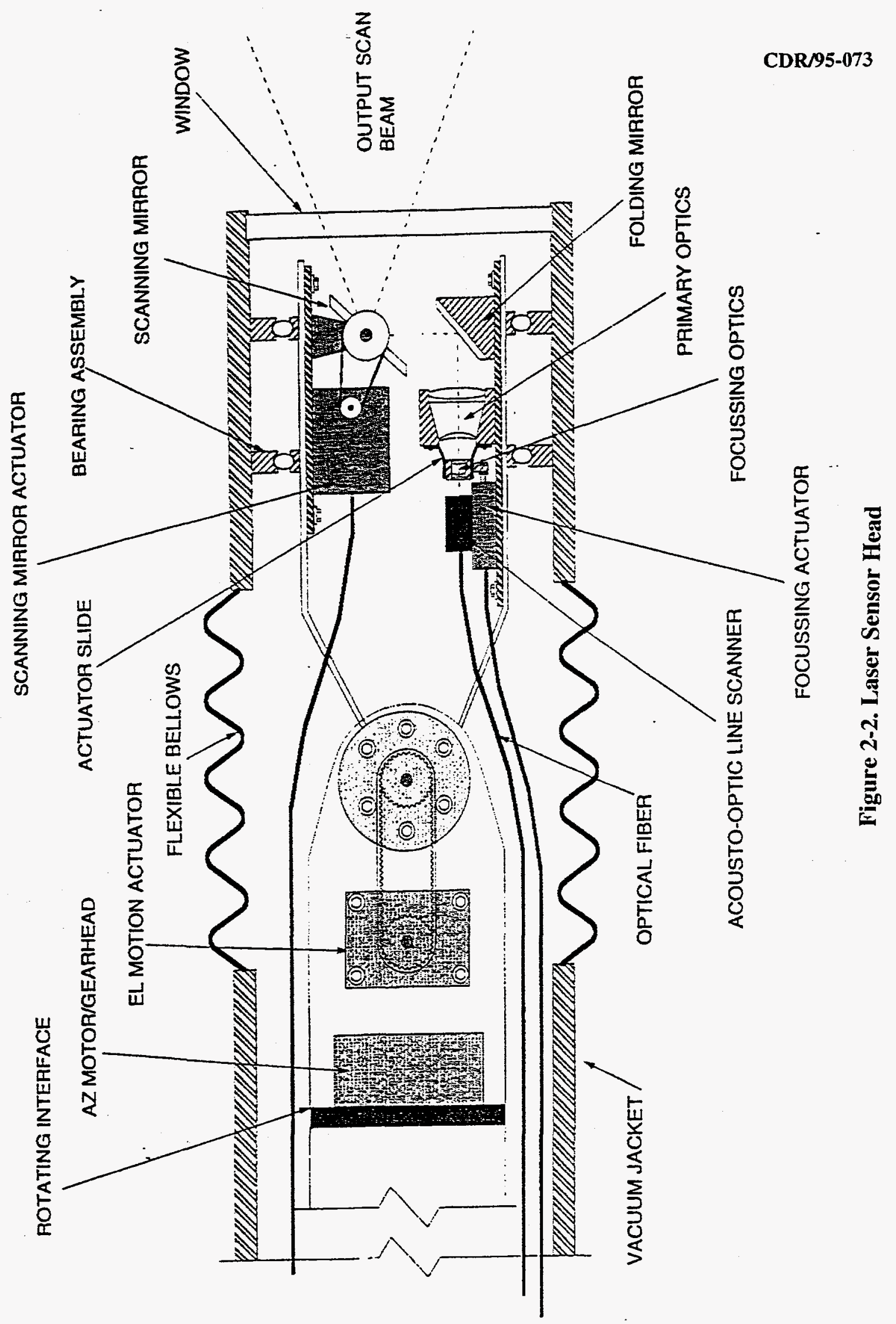


is almost straight down in order to avoid exerting torque on the vacuum jacket. The bearing on the end section of the vacuum jacket fixes the position of the scanner with respect to the window so that the window refraction effects can be calibrated.

For any given position of the two arm joints, the scanner will be capable of making measurements within an approximately a $30 \times 30$ degree field of view. In order to cover the entire spherical field of view, the scanner will be repositioned using the probe arm. One measuring scenario is to scan successive $30 \times 30$ degree fields along an elevation circle (12 positions along the 360 degrees), increment the azimuth angle by 30 degrees, and repeat the elevation position measurements. Oversampled regions need not be measured again. Once the azimuth angle has been incremented by a total of 180 degrees, the entire volume has been scanned. Alternately the total elevation angle can be 180 degrees per measurement set with a total azimuthal scan of 360 degrees.

The extended length of the RMS measurement boom may cause stability and pointing accuracy problems at the window end. Because of this, it will be necessary to have fiducial reference points within the reactor or to perform a post measurement data fit to assist in fitting together the 30 $x 30$ degree view regions. At a $1 \mathrm{~mm}$ positional uncertainty at a range of $10 \mathrm{~m}$, this translates to a pointing accuracy of $\arctan (.001 / 10)$ or .0057 degrees (21 arcsec). It is difficult to imagine the RMS probe inserted and positioned with the required accuracy to achieve this.

For the scanner assembly itself, we have considered three options, a completely mechanical approach using galvanometers, an acousto-optical (AO) scanner, and a hybrid approach.

\section{The All Mechanical Scanner Approach}

A mechanical approach using a galvanometer or a similar moving mirror system has the advantage of being fairly simple and straight forward to implement and of being rugged enough to survive the environment. Good galvanometers have angular repeatability values of about 10-30 urad (.00057 to .0017 degrees) which are good enough for this application. Scanning speed will depend upon several factors including mirror size but a $10 \mathrm{msec}$ settling time is achieved by units such as those provided by Cambridge Instruments. The principal concern with an all mechanical system is measurement speed and this measurement speed is limited by doppler noise. We consider the mapping of the ITER interior surface to be equivalent to mapping a sphere with a $10 \mathrm{~m}$ radius. At a 1 point $/ \mathrm{cm}^{2}$ density there are $1.26 \times 10^{7}$ measurement points. If six measurement stations are used there are $2.09 \times 10^{6}$ measurements/station. If eight hours are used by each station for a measurement run the measurement speed must be at least 73 measurements/second. Allowing for time to reposition the probe head, 100 measurements/second becomes a reasonable minimum measurement rate for mapping the interior of the ITER plasma chamber on a $1 \mathrm{~cm}$ grid.

At a range of $4 \mathrm{~m}$ a beam moving at $1 \mathrm{~cm} /(0.01 \mathrm{sec})$ requires an angular velocity of 0.125 radians/sec by the scanner mirror. With a $1 \mathrm{~cm}$ aperture this implies a maximum differential surface 
velocity of $1.25 \times 10^{3} \mathrm{~m} / \mathrm{sec}$. For laser light at a wavelength $\lambda=1.5 \mu$, this leads to a frequency uncertainty of $1.67 \mathrm{kHz}$. For a FMCW laser radar sweeping $100 \mathrm{GHz}$ in $1 \mathrm{msec}$ the relationship between frequency and range is approximately $600 \mathrm{Khz} / \mathrm{m}$ or $600 \mathrm{~Hz} / \mathrm{mm}$. Thus, this maximum doppler noise corresponds to a range error of $2.8 \mathrm{~mm}$, which is not tolerable.

The doppler noise from an all mechanical scanner can be mitigated by adding a piezoelectric counter scanner which comes close to freezing the beam position during the measurement dwell period. This type of scanner was successfully developed and delivered to NASA as part of a 3D vision system in 1987. The complexity of this approach reduces its attractiveness, but it remains an alternative approach should the high $\gamma$ radiation level testing of AO components not confirm our expection of their ability to perform in the ITER environment.

The precision mechanical nodding mirror components available from Cambridge Instruments have a maximum temperature rating of $150^{\circ} \mathrm{C}$ for the non permanent magnet units which have the highest temperature tolerance. Either a more tolerant mirror motor must be found or temperature within the shell of the RMS probe will have to be maintained sufficiently below $150^{\circ} \mathrm{C}$ to assure reliable operation.

\section{The AO Scanner Approach}

An AO scanner has the advantage of no uncorrectable doppler effects combined with high speed operation. However, an AO scanner has optical signal losses because of Bragg cell diffraction inefficiency. Compared with a one dimensional Bragg cell beam deflector, a two dimensional device will have more than twice the losses due to the diffraction inefficiency, because of design constraints in a two stage beam deflection. In addition, the two dimensional AO scanner will involve two Bragg crystals and twice as much optical absorption induced by $\gamma$ radiation damage. Thus, although a no moving parts 2D AO scanner is extremely attractive because of its speed and random pixel addressability, at this point it is prudent to pursue a hybrid mechanical/AO scanner.

\section{The Hybrid AO/Mechanical Scanner Approach}

A hybrid approach consists of a 1-D AO scanner with a nodding galvo mirror to supply motion in the other direction. The hybrid approach uses the AO beam deflector to move the beam in the fast scanning direction. Beam motion in the mechanical mirror steered beam direction is sufficiently slow that doppler induced measurement noise is not significant. At the same time, the AO scan in the orthogonal direction easily support a 1000 measurement/second rate which is probably a near optimum tradeoff between speed and accuracy for this application. For these reasons the hybrid $\mathrm{AO} /$ mechanical scanner will be pursued as the preferred option, maximizing performance at acceptable technical risk. 


\section{Scanner Focus}

Independent of the scanner approach is the question of focusing. A fix focus system is appealing in its simplicity and, with sufficient power, could be practical. However, a focused system provides substantially superior signal-to-noise for the receiver to process and a smaller beam footprint which leads to more accurate measurements, particularly on sloped surfaces. A focused system will operate substantially slower because of the additional time required for range search if range is not approximately known beforehand or is not trackable. For the ITER 3D mapping operation surfaces will be relatively smooth and generally continuous. In addition, the approximate range will be known beforehand so that focus position can be based on the pointing coordinates. Therefore, focusing can be accomplished with little or no impact on measurement speed and with a substantial improvement in range measurement signal quality and range accuracy.

\subsection{RMS Concept of Operation}

The RMS probes will be deployed through at least six port locations located on the top of the reactor vessel directly above the divertor. The probe will descend sequentially to a set of fixed levels. At each of these levels the end of the RMS probe will rotate through a series of fixed positions in azimuth and elevation. At each position the RMS scanner will direct the CLR laser beam to those surface points which are best mapped from that position, plus additional fiducial points and overlap points which are useful for integrating the total collection of scans into single reference frame 3D map of the interior of the reactor vessel.

By this systematic rigid approach to reactor 3D mapping, speed is optimized and the gathered data is most readily compared with previous collections in an analysis of changes. The high speed of the survey results from all measurement parameters such as focus, being approximately known ahead of time. This speed minimizes exposure of the RMS probes to the ITER radiation environment. A total surface map from one probe position will take one hour or less at 1000 measurement/sec. If additional specialized runs are necessary, for example, a high density scan of a small surface region, the entire operation will be planned and its drive parameters precomputed prior to probe deployment..

\subsection{RMS Measurement Performance}

\subsubsection{Range Measurement}

Measurement speed and required measurement density are driving factors for exposure of the RMS scanner probe to the high $\gamma$ radiation and high temperature environment. Although the 3D measurement operation may begin with specialized slower scanning to locate fiducial slopes and thus establish the position of the scanner head, the balk of the 3D mapping operation will be occupied scanning a one $\mathrm{cm}$ grid over the reactors internal surface. The area of that surface is about that of 
a sphere with a $10 \mathrm{~m}$ radius, requiring $1.26 \times 10^{7}$ measurements. If this mapping task is divided between scanners located in each of six sections of the ITER, the mapping of each quadrant may be completed in less than an hour at a measurement rate of $1000 / \mathrm{sec}$. With a 256 point linear lenslet array addressed by the $\mathrm{AO}$ beam deflector, the linear array will be scanned four times per second while the nodding minor was in continuous slow motion, moving the target point $0.04 \mathrm{~mm}$ during the single measurement dwell. The 1000 measurement/second rate is accomplished by the current CLR 3D mapper produced by CRC. The coherent laser vision system (CLVS) under development will perform measurements at sixteen times that rate. The theoretical range measurement accuracy obtainable for a set of dwell times is given in Table 2-1, as derived in Appendix A. These range measurement error estimates assume a $6 \mathrm{~dB}$ round trip signal loss in the beam deflection Bragg cell caused by the maximum expected radiation darkening, as discussed in Section 2.4.2. The assumed laser power is only $1.0 \mathrm{~mW}$. This power may be boosted to $100 \mathrm{mw}$ using a fiber optic amplifier to overcome any other sources of radiation induced signal loss, as in the optical fiber.

\begin{tabular}{|c|c|}
\hline Measurement Dwell Time T & Range Error $\sigma_{R}$ \\
\hline $10^{-4} \mathrm{sec}$ & $0.07 \mathrm{~mm}$ \\
\hline $10^{-3} \mathrm{sec}$ & $0.03 \mathrm{~mm}$ \\
\hline $10^{-2} \mathrm{sec}$ & $0.007 \mathrm{~mm}$ \\
\hline
\end{tabular}

Clearly, the required range measurement accuracy of $0.1 \mathrm{~mm}$ should be achievable by the CLR. A greater contributor to 3D measurement error will probably be the location of the scanner head position and calibration and correction for thermally induced changes in scanner orientation and position.

\subsubsection{Angle Measurement}

For a given lenslet position and a given galvanometer mirror encoder reading actual laser beam heading will be a function of temperature. It is known ${ }^{5}$ that radiation induced heating affects the Bragg cell beam deflection. The Bragg cell acoustic drive frequencies will be temperature adjusted to ensure that the deflected beam positions continue to center on the individual lenslets. However, there will be an expansion of the lenslet array itself with temperature which will cause significant beam deflection. For example, for a $100^{\circ} \mathrm{C}$ temperature change and a $1.0 \times 10^{-5}$ coefficient of thermal expansion for the lenslet array, (Borosilicate crown glass) the linear array end laser beam spot at $4 \mathrm{~m}$ range will move approximately $1.4 \mathrm{~mm}$. There will be other effects from change in index of refraction of the lens system and from thermal expansion of the support structure. Although these effects may be estimated theoretically and the projected effects used to select 
materials and structure design, the completed scanning system will need to be calibrated empirically as a function of temperature. At run time, one or more temperature sensors must be monitored in order to make appropriate adjustments to the Bragg cell drive frequencies and to the estimated scan angles.

A large potential source of 3D measurement error stems from the positioning of the scanner head. The $10 \mathrm{~m}$ long deployment boom will inevitably place the scanner head in an uncertain position because of both thermal and mechanical effects. Therefore, each time the boom elevation is adjusted to scan a new section of the ITER interior, the position of the scanner must be reestablished. If sharp corners or edges can be seen from two or more boom elevations, observations of these features may be used to establish the relative 3D transformation between the two or more data sets. If not, a least squares fit may be used encompassing all of the thousands of points from the overtap region of adjacent 3D mapped sections of the reactor interior. Since the data points from two sets generally will not be coincident, matching the two sets will involve curve fitting and interpolation. Because of large numbers of measurement points involved, the method should still be quite accurate. The effectiveness of using discrete surface artifacts, (reflective features, seams, holes, peaks, corners, cracks, edges) will depend upon their existence, either man made or by the weathering action of the reactor's operation. The second procedure, more statistical in nature than one based upon recognition of specific features, will also depend upon naturally occurring surface variations unless it is permitted to introduce variations in surface shape or reflectivity.

In order to determine the potential effectiveness of either method for orienting the scanner within the reactor, it might be useful to perform a precision 3D mapping of an existing fusion reactor such as the Princeton Tokomak in order to see what measurable surface detail exists.

\subsection{System Components}

\subsubsection{Fiber Optic Circuitry}

The measurement arm fiber optic circuitry for the RMS with polarization maintaining (PM) optical fiber is shown in the system block diagram as shown in Figure 2-1. This is the basic optical circuitry which is used in the CLR precision 3D mapper currently produced by CRC. The reference arm branch is left out of the figure for simplicity. PM fibers maintain a constant polarization relationship between the optical signals in the different paths such as the local oscillator path and the target path in Figure 2-1. Because of the delayed local oscillator circuits, this current configuration will be effective to at least $30 \mathrm{~m}$ range.

An alternative fiber optic circuitry for the RMS, which may be implemented with single mode (SM) optical fiber, is shown in the system block diagram in Figure 2-3. This optical circuit approach for RMS is the same as was used by CRC on a multiprobe CLR system which was delivered to NASA. With this circuit the reflection off the end of the fiber serves as the local 


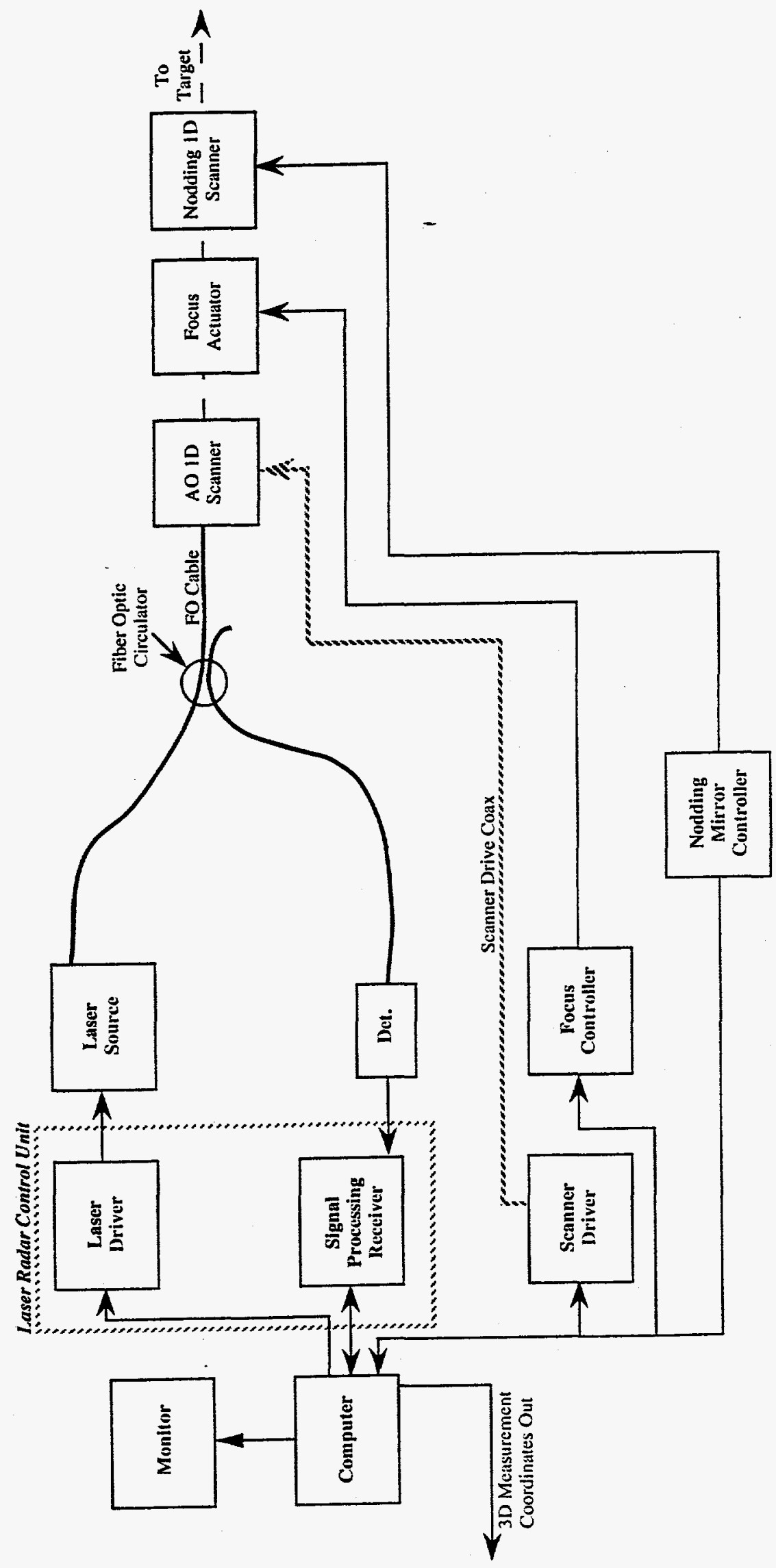

CDR/95-073

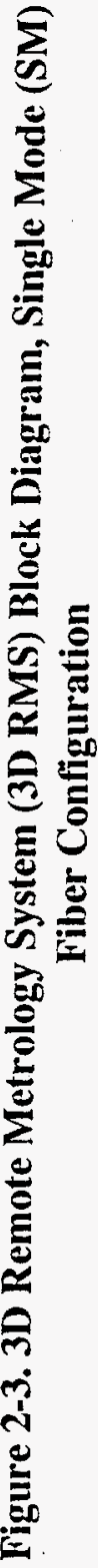


oscillator signal and mixes with the target return signal at the point of the end of the fiber. As long as the path from the end of the fiber to the target and back does not exceed the coherence length of the laser diode output, this circuit approach will be effective. The new laser diode type received from GEC Marconi will support a measurement range of at least $10 \mathrm{~m}$ with this circuit approach. If the RMS deployment scheme with the long probe arm is used, as in Figure 2-2, and if probes are deployed from four or more measurement plots, this should be adequate range to map the entire interior chamber of the ITER.

Multiple sources have reported that polarization maintaining fibers are more susceptible to ionizing radiation than pure silica core single mode fiber ${ }^{7,8,9,10}$ Tests with rad resistant PM fiber have attenuation less than $10 \mathrm{~dB} / \mathrm{km}$ for $\gamma$ radiation doses as large as $10^{5} \mathrm{rads}^{11}$. Although these fibers have not been tested at the multiple Mrad level required for ITER, it is noticed that the attenuation vs. dose levels out near $10^{5}$ rads.

Figure 2-4 (from reference 12) shows that $\gamma$ radiation induced attenuation in a pure silica core single mode fiber at radiation levels to $10^{8} \mathrm{rad}$. These tests were for an optical wavelength of $1.3 \mu$. At the $1.5 \mu$ wavelength used by the RMS, the attenuation will be less. For the reported tests after one hundred hours exposure to $\gamma$ radiation at the $10^{6} \mathrm{rad}$ per hour level the optical fiber attenuation is only $100 \mathrm{~dB} / \mathrm{km}$ or $2 \mathrm{~dB}$ for the approximately $20 \mathrm{~m}$ exposed fiber round trip path length in the ITER RMS probe. Clearly, the single mode fiber approach is a solution in hand for the RMS system configuration. There may be some small additional losses at the Bragg cell because of the inability to align the polarization for optimal device efficiency, but these losses will not cause significant performance degradation.

Because of the PM fiber approach's performance advantage of unlimited range for ITER chamber 3D mapping, $\gamma$ irradiation tests should be performed to the $10^{8} \mathrm{rad}$ level with the most rad hard PM fiber obtainable. These tests will determine whether these PM fibers will survive the ITER environment with an acceptable attenuation level. These tests should be performed with the fibers at a temperature of $100^{\circ} \mathrm{C}$ to take advantage of the beneficial annealing which will occur at the elevated temperature ${ }^{2}$ in the ITER chamber. Without a successful PM fiber radiation test, the single mode approach will remain the choice.

\subsubsection{Acoustooptic Bragg Cell}

An acoustooptic Bragg cell is used for beam deflection in the first stage of a two dimensional scanner (see Figure 2-2). The second stage is a mechanical nodding mirror. The Bragg cell accomplishes beam deflection by having an acoustic wave create a spatial modulation of the index of refraction which serves as a diffraction grating. When the acoustic frequency is shifted, the grating line spacing and beam deflection angle change accordingly. Since the $1.5 \mu$ laser light passes through the Bragg cell twice, minimizing signal loss for each pass is extremely important. 


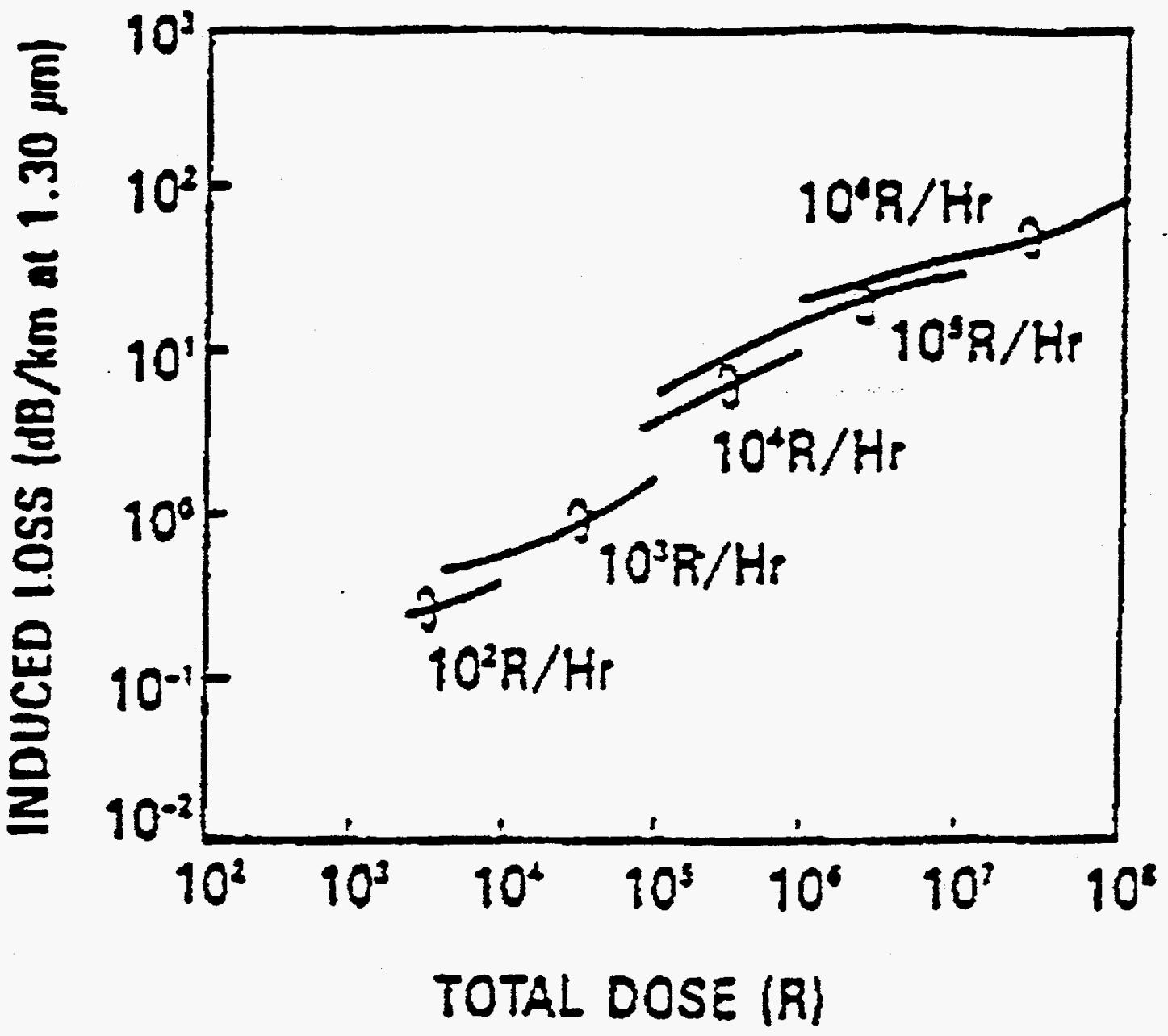

Figure 2-4. $\gamma$-ray Irradiation Characteristics 
Candidate materials for Bragg cell crystals include $\mathrm{TeO}_{2}, \mathrm{GaP}, \mathrm{InP}$, or $\mathrm{PbMnO}_{2}$. Of these $\mathrm{TeO}_{2}$ has by far the greatest Bragg efficiency (puts the most energy into the deflected beam).

Bragg efficiencies of nearly $80 \%$ are achievable with $\mathrm{TeO}_{2}$ (2-3dB two way insertion loss) driven 5 watts of RF power to generate the acoustic wave. By contrast, $\mathrm{GaP}$, which is more radiation resistant, has only $1 \%$ of the Bragg efficiency of $\mathrm{TeO}_{2}$. Driving a GaP Bragg cell with the same power will result in about $23 \mathrm{~dB}$ two way insertion loss. Driving the $\mathrm{GaP}$ cell significantly harder to achieve higher Bragg efficiency will likely cause thermal damage to the crystal.

On the other hand, $\mathrm{GaP}$ is the Bragg cell material least vulnerable to radiation induced optical signal losses and $\mathrm{TeO}_{2}$ is significantly more vulnerable. However, even at the $10^{6} \mathrm{Rad}$ level of radiation the one way $\mathrm{TeO}_{2}$ Bragg cell loss will be less than $3 \mathrm{~dB}$ (private communication 1.995, Edward Taylor, Phillips Lab, Albuquerque, NM). More test results are required to determine the actual alternation level of a continuous $3 \times 10^{6} / \mathrm{hr}$ dose as a function of time. It is attractive to stay with a Bragg cell material which has fundamentally better performance parameters if the expected radiation-induced loses can be tolerated.

A second effect of high radiation doses is a variation of beam deflection which is thought to be caused by radiation-induced heating of the AO crystal.

Operationally, it will be important to operate the Bragg cell at constant RF drive power in order to maintain thermal equilibrium. The RF drive frequencies will have to be adjusted for the expected thermal offset to obtain the correct beam deflection. Tables of appropriate RF drive frequencies as a function of temperature will be maintained for each lenslet position.

\subsubsection{Lenslet Array}

The lenslet array serves as target for the AO Bragg cell beam deflector. The illuminated lenslet spreads the laser beam to fill the aperture of the lens system which them focuses the beam on the scene position corresponding to a pixel in the CLVS image. The lenslet array is like camera film at the image focal plane of the lens system. In the case of the RMS only one dimension is scanned using a linear lenslet array. The second dimension of scanning is accomplished by a mechanical nodding mirror. The fast scanning will be done in the direction of the lenslet array, slower orthogonal scanning by the nodding mirror.

Fast scanning in two dimensions may be accomplished by using two orthogonal Bragg cells in sequence, followed by a rectangular lenslet array. This configuration is used by the coherent laser vision system (CLVS) being developed by Coleman Research for DOE. However, the addition of radiation induced optical absorption to the transmission inefficiencies of the double Bragg cell makes the 2D AO scanner risky until both of these factors have been determined quantitatively and verified experimentally. The 2D Bragg cell efficiency will be verified in early 1996 by the CLVS program 
at CRC. The radiation induced optical losses in the Bragg cell should be determined in 1996 through continuing work by Edward Taylor's group at Phillips Lab, particularly if coordination with ORNL assures that the requisite measurements and analysis take place. In the mean time, a single axis $\mathrm{AO}$ beam deflector will have half the radiation loss and less than half the loss through transmission inefficiency and will provide the increase in speed and reduction in doppler noise necessary to accomplish the RMS fast, accurate 3D mapping mission.

The lenslet array is prepared by etching either refractive or diffractive lens shapes onto a sheet of glass. For the RMS the glass sheet thickness will be approximately $0.4 \mathrm{~mm}$ and each lenslet will be approximately $0.125 \mathrm{~mm}$ in diameter. The lenslet arrays can be made of rad hard glass such as Schott $\mathrm{Bk} 7$ or from quartz ${ }^{1}$. Even at the $10^{\circ}$ rad irradiation level, a lenslet array should not deteriorate significantly in transmission performance.

\subsubsection{Lens System Optics}

Glasses are available for lenses which show minimal degradation at the $\gamma$ radiation doses expected from ITER. For example ${ }^{3}$, purified fused silica (corning 7940) shows only an $11 \%$ degradation intransmission at $\lambda=0.7 \mu$ and below after a dose of $5 \times 10^{9} \mathrm{R}$. For all glasses considered radiation induced reduction in transmission decreases with wavelength. Therefore, we expect that at $\lambda=1.5 \mu, 1000$ hours of operation in a $3 \times 10^{6} \mathrm{R} / \mathrm{hr}$ environment can easily be tolerated by glass components.

Antireflective (AR) coatings are used on lenses to maximize transmission and reduce interference from reflected signal components. Given sufficient laser power it will probably be possible to operate without AR coatings. Data on previous radiation test of AR coatings or new tests with AR coatings would be obtained.

\subsubsection{Metal Mirrors}

Metal mirrors have been shown to survive and perform after direct exposure to $\gamma$ and neutron radiation $\left(10^{2 i} \mathrm{n} / \mathrm{cm}^{2}\right)^{(4)}$ of an active reactor. Clearly, these mirrors should survive the $3 \times 10^{6} \mathrm{R} / \mathrm{hr}$ levels expected during ITER 3D mapping. With a configuration of metal mirrors and baffels, glass window and shielded fiber, it would be possible to perform mapping in a higher radiation environment than the planned ITER post operation scenario.

${ }^{1}$ Private communications, Timothy Patterson, Teledyne Brown, manufacturer of lenslet arrays for the CLVS scanner. Optical transmission efficiency for $\lambda=1.5 \mu$ is expected to be significantly better than $99.9 \%$ at the $10^{8}$ rad level, assuming that a longer, more transmitting wavelength will perform as well as $\lambda=0.7 \mu$. A lenslet array made with a quartz sheet should have an internal transmission efficiency better than $97 \%$ after a $10^{9}$ rad irradiation. 


\subsubsection{Motors and Bearings, Mechanical Joints}

The RMS design approach encloses the joints, drive motors for those joints and encoders/resolvers in the pressurized RMS probe vessel. This enclosure may also permit the interior of the probe vessel to be cooled via an air stream introduced through the metal bellows enclosed umbilical which connects the atmospheric pressurized probe vessel with the chamber exterior environment.

Motors are available which withstand $10^{8}-10^{9}$ rad $\gamma$ radiation levels ${ }^{3}$. The maximum temperatures tolerated by these motors is undetermined. Optical encoders are claimed to be "rad hard" and with a temperature tolerance of $300^{\circ} \mathrm{C}^{13}$. However, these properties have not been tested. It makes sense to make use of robotic joints designed for DOE or nuclear industry use which can withstand the radiation and temperature environment expected in ITER. The component examples indicate that such joints should be produceable. If they already exist they must be tested under ITER conditions. If not they will be needed for the whole range of ITER chamber operations, as well as the RMS, and must be developed.

The RMS enclosed scanner configuration at least relaxes the high vacuum requirement for joints, joint drive motors, and encoders. Lubricants exist which withstand $3 \times 10^{9} \mathrm{rad}$ levels but stated maximum temperature tolerance is $163^{\circ} \mathrm{C}$ (NLGI Grade $2 \frac{1}{2}$, synthetic arouatic oil; sodium omate thickness; Delenid oxidation modulator) ${ }^{3}$. Although contamination of the high vacuum ITER chamber is not a concern because of the enclosure of the RMS probe, there may be need for concern about clouding of optical components by exposed, heated lubricants. Again, positive air flow from the bottom of the probe to the top will aid this situation.

Since the typical maximum temperature ratings for a wide range of components and lubricants is below the $200^{\circ} \mathrm{C}$ ambient temperature of the ITER chamber, it makes sense to cool the interior of the RMS probe vessel. The effectiveness of such a cooling scheme should be tested prior to setting the component temperature tolerance requirements.

The drive mechanism for inserting and withdrawing the RMS probe will necessarily have to withstand the high vacuum environment and at least some of the radiation and high temperature conditions. The design of this mechanism is beyond the scope of this study.

\subsubsection{Electrical Cables and Wires}

Electrical cables are available ${ }^{3}$ radiation resistant at levels $\left(6 \times 10^{14} \mathrm{R}\right)$ far above the requirements for 3D RMS. For the design concept development it will be necessary to verify that the insulating materials used in these cables are compatible with a high vacuum at $200^{\circ} \mathrm{C}$ temperatures. 


\subsubsection{Metal Structural Parts}

Most properties of metals are not significantly affected by gamma irradiation and metals are the preferred material for radiation resistance. Damage threshold levels above $10^{12}$ or $10^{13}$ rad apply for most metal ${ }^{3}$.

\subsubsection{Temperature Sensor}

One or more temperature sensors will be necessary to temperature correct Bragg cell drive frequencies to compensate for expansion of structural and optical components, and to insure that provided cooling is adequate to protect temperature vulnerable components. Rad hard temperature sensors are not among the sensors discusses in the literature surveyed. It is assumed that they exist for nuclear industry and DOE applications.

\subsection{Alternative RMS Design Approach (Switched Fiber Optic Scanner)}

The RMS design approaches discussed so far include some combination of Bragg cell beam deflection and mechanical scanning. The indications are that a Bragg cell will be able to survive the $\gamma$ radiation environment and provide adequate transmission efficiency. If further testing proves this not to be true and if the speeds of a purely mechanical 2-dimensional scanner are too slow, then the scanner approach we will now discuss may be of interest.

The alternative design replaces the lenslet array with an array of fiber ends as the target for the $\mathrm{AO}$ beam deflector. Instead of 128 or 256 lenslets in a row, there are 128 or 256 optical fiber ends. The Bragg cell beam deflector is located in a low radiation environment. A bundle of fibers then connects the beam deflector to an output array of fiber ends in the optical system at the end of the RMS probe. This array of fiber ends serves essentially the same purpose as the lenslet array in the approach discussed above. The reward for this approach is the removal of the last potentially radiation vulnerable optical component from the RMS probe and avoidance of Bragg cell radiation absorption losses. The penalty for this approach is greater complexity, the need for a bundle of fibers rather than a single fiber down to the end of the RMS probe, and losses coupling from the AO deflector into the fiber array. This approach should be considered if further Bragg cell radiation tests are not positive. 


\subsection{Conclusion and Recommendation}

RMS requirements will be met, but further radiation and temperature testing are needed to obtain the most robust and cost effective approach. Radiation tests to the $10^{8} \mathrm{rad}$ level should be performed for $\mathrm{TeO}_{2}$ Bragg cells and for $1.5 \mu \mathrm{PM}$ fibers ${ }^{2}$. These tests should performed at $100^{\circ} \mathrm{C}$ to take advantage of the annealing effect of the ITER interior temperature, possibly cooled in the RMS probe interior. A mockup of the RMS probe should be built and insulation and cooling schemes should be tested to determine what temperature reduction can be relied upon in the interior of the RMS probe. Surveys for almost all electromechanical components and lubricants revealed that $200^{\circ} \mathrm{C}$ is well above the rated operating range. Meeting radiation tolerance requirements is less of a problem. If a temperature below $150^{\circ} \mathrm{C}$ can be maintained, many components become suitable including precision mechanical $1 \mathrm{D}$ scanner mirror drivers.

With this testing complete major design option decisions can be made and the detailed RMS system design can proceed.

${ }^{2}$ Edward Taylor at Phillips lab is interested in performing these tests. 


\subsection{REFERENCES}

1. A.T. Ramsey, D-T Radiation Effects on TFTR Diagnostics, Princeton Plasma Physics Laboratory, Princeton University, Princeton, New Jersey, October 1994.

2. J.K. Partin, J.O. Bostad, D.R. Collins, Real-Time Attenuation of Heated Optical Fibers in a Nuclear Reactor, Photon ‘83, EG\&G Idaho, May 16-19, 1983.

3. K.U. Vandergriff, Designing Equipment for Use in Gamma Radiation Environments, Oak Ridge National Laboratory, Martin Marietta, May 1990.

4. D.V. Orlinski, Radiation Effects on In-Vessel Components, ITER Workshop on Nuelear Materials, Max Planck Institute, Garshing bei Munchen, 1993.

5. E.W. Taylor, A.D. Sanchez, S.A. Dewalt, R.J. Padden, S.P. Chapman, T.W. Monarski, D.M. Craig and D.J. Page, Radiation Induced Effects in Acousto-Optic Devices, AFMC Phillips Laboratory, Paper Presented at: The International Society for Optical Engineering OE/Fibers '92 Symposium, August 1992.

6. R.A. Greenwell, D.M. Scott, and J.J. McAlarney, Nuclear Survivable Polarization Fibers for Fiber Gyroscopes on Spacecraft, Oak Ridge National Laboratory, Presented at SPIE OE/fibers Symposium, Boston, MA September 1992, August 1992.

7. Scott, D.M., C.E. Barnes, and R.A. Greenwell, Gamma-induced Attenuation in PolarizationMaintaining and Single Mode Fibers, Fiber Optics Reliability: Benign and Adverse Environments III, SPIE Proceedings 1174-04, pages 33-37, 5-7 Sept. 1989, Boston, MA.

8. Friebele, E.J., M.E. Gingerich, L.A. Brambani, C.C. Harrington, and S.J. Hickey, Radiation Effects in Polarization Maintaining Fibers, Fiber Optics '90, SPIE Proceedings 1314-21, 24-26 Apr. 1990, London, UK.

9. Brambani, L.A., E.J. Friebele, C.G. Askins, M.E. Gingerich, and J.R. Onstott, Radiation Effects in Polarization Maintaining Fibers, Fiber Optics Reliability: Benign and Adverse Environments II, SPIE Proceedings 992-07, pages 43-49, 6-8 Sept. 1988, Boston, MA.

10. Friebele, E.J. L.A. Brambani, M.E. Gingerich, S.J. Hickey, and J.R. Onstott, Radiation Induced Attenuation in Polarization Maintaining Fibers: Low Dose Rate Response, Stress, and Materials Effects, Applied Optics, pages 5138-5143, Vol 28, No. 23, 1 Dec. 1989.

11. M.E. Gingerich, D.L. Grission, E.J. Frebele, Degradation of Optical Fiber in the Nation Space Environment. Presented at OE/Aerospace Science and Sensing, 1993. 
12. Y. Chiguse etal, $\gamma$ Ray and Neutron Irradiation Characteristics of Pure Silica Cor Single Mode Fiber and its Life Time Estimation, IEEE Transactions on Nuclear Science, Vol 35, No. 1, February 1988.

14. R.E. Barry, M.M. Menon, and A. Slotwinski, A $3 D$ Remote Metrology System for the International Thermonuclear Experimental Reactor, paper to be submitted, Oak Ridge National Laboratory, Martin Marietta, 1994. 
APPENDIX A

Performance Estimate for the ITER RMS, a Fiber Optic FMCW Coherent Laser Radar 


\section{A.1 System Description}

The fiber optic coupled FMCW coherent laser radar uses the relatively large tuning range of injection laser diodes to achieve greater precision than available with other techniques. As shown in Figure A-1, the optical frequency of the laser is swept linearly as a function of time. The laser output is divided and used both as a local oscillator (L.O.) And as the signal to be transmitted. After being time delayed by the round trip transit time to the target, the received signal is mixed with the optical L.O. on a photodetector. The resultant beat frequency is equal to the sweep rate of the optical signal multiplied by the time delay between the received signal and the local oscillator. Since this time delay is proportional to target distance, the RF beat frequency is also proportional to target distance.
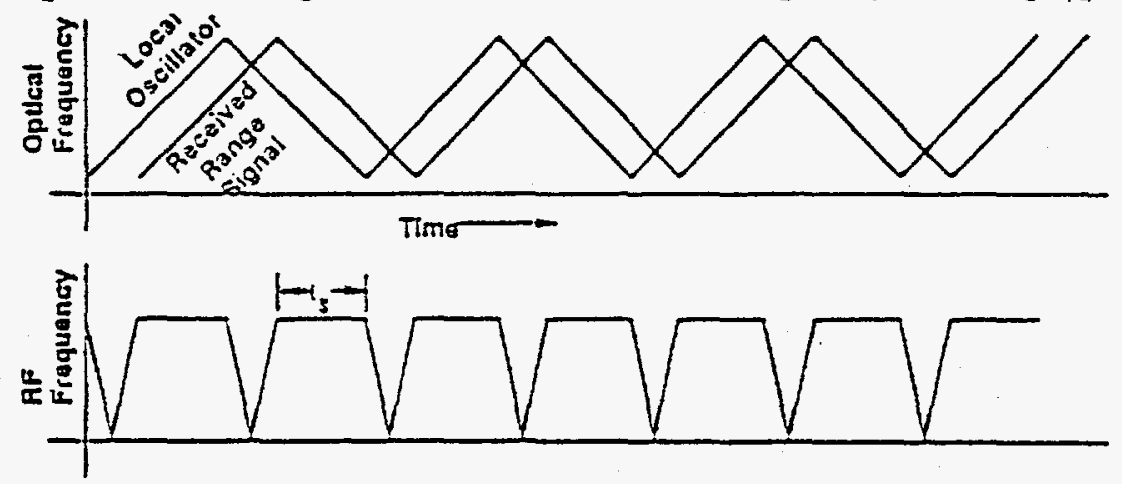

Figure A-1. Laser optical frequency and heterodyned RF SIGNAL Coherent Laser Radar

Due to the short wavelength of the laser compared to a microwave source, a proportionally small frequency deviation results in a large beat frequency. For example, at an optical wavelength of $1550 \mathrm{~nm}$, a shift in wavelength of only 1 Angstrom $(0.1 \mathrm{~nm})$ results in a frequency shift of 12 GHz. This frequency modulation is accomplished by modulating the laser's injection current thereby thermally tuning the laser wavelength. The sensing system optical configuration is shown in Figure A-2.

The FMCW coherent laser radar's source consists of a $1550 \mathrm{~nm}$ single mode diode laser pigtailed to an optical fiber. The laser's optical output is frequency modulated by varying its injection current. In the basic configuration shown, one half of the output light is directed into the L.O. path via a $3 \mathrm{~dB}$ coupler. The remainder of the light is focussed by the antenna lens into the range measurement area of interest. Light reflected from a surface in this area is recollected by the lens, split into the return signal path and mixed with the L.O. light at the second $3 \mathrm{~dB}$ coupler. By utilizing two detectors and a differential amplifier common mode rejection of amplitude noise is achieved. Signal loss due to coherence length limitations of the source can be compensated for by adjusting the L.O. path length such that it and the measurement path are approximately of equal length. 


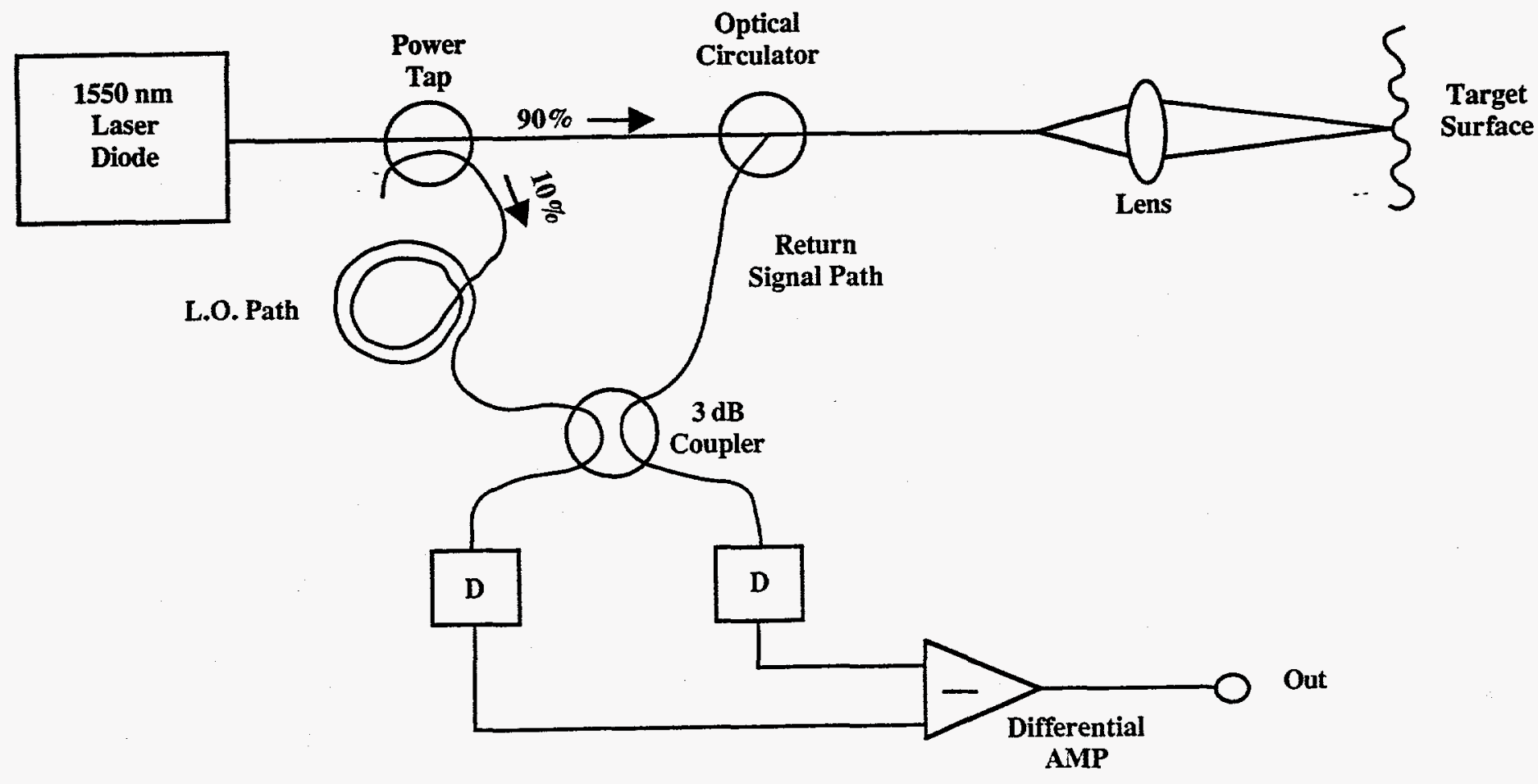

Figure A-2. Basic Fiber Optic FMCW CLR Configuration 


\section{A.1 CLR SYSTEM SNR}

The signal generated by the FMCW CLR in the RMS is proportional to the amount of light reflected off the target and recaptured by the antenna lens. Thus, the signal depends on the target surface's roughness and absorption properties. In a radar system, the target characteristics are represented in a term called "target cross section" which is defined as follows:

Target Cross Section $=\frac{4 \pi \text { Power Reflected Per Unit Solid Angle }}{\text { Incident Power Per Unit Area }}$

For an opaque solid composed of a number of individual scatterers that are the order of the wavelength of light, the scattered field is Lambertian where the reflected light is confined to a solid angle of $2 \pi$ steradians oriented normal to the surface. given by:

The ratio of the energy intercepted by the antenna lens to the energy incident of the target is

$$
\text { ratio }=\frac{\frac{\pi}{4} d^{2}}{2 \pi R^{2}}=\frac{\rho d^{2}}{8 R^{2}}
$$

where $d=$ lens aperture diameter

$\mathrm{R}=$ range to target

$\rho=$ ratio of reflected to incident light on target

Equation (A.1) incorporates the target reflectivity, $\rho$, and the space loss. A third term, transmission loss $\left(\mathrm{L}_{t}\right)$, must also be included. In general, transmission loss is defined as the fractional amount of optical energy that remains after the light is attenuated by such factors as coupler splitting ratios, lens component reflections, and atmospheric attenuation. Due to the short range application of this sensor, atmospheric effects can be ignored and the losses associated with the system components dominate. If $\mathrm{P}_{\mathrm{t}}$ is the power transmitted by the laser source, the signal power, $P_{s}$ that returns to the laser is given by:

$$
P_{s}=\frac{P_{t} \rho L_{t} d^{2}}{8 R^{2}}
$$

where $L_{t}$ is the two-way transmission loss factor. 
In any optical system, the minimum detectable, or quantum limited, power, $\mathrm{P}_{\mathrm{Q}}$ is given by:

$$
P_{Q}=\frac{h v B}{\eta}
$$

where $\mathrm{h}=$ Planks constant

$v=$ Optical frequency

$\mathrm{B}=$ Measurement bandwidth

$\eta=$ Detector quantum efficiency

The condition for quantum limited operation of heterodyne receiver is that the shot noise produced by the local oscillator (L.O) in the detector is sufficient to override the thermal noise in the amplifier. If the current generated by the L.O. is much greater than both the photodetector dark current and the signal current, then the heterodyne noise equivalent power is given by:

$$
(N E P)_{H e t}=\left[\frac{h \eta}{v}+\frac{2 k T}{P_{L O}}\left(\frac{h \eta}{v e}\right)^{2} \frac{1}{R}\right]
$$

where $\mathrm{k}=$ Boltzman constant

$\mathrm{T}=$ Effective amplifier temperature

$\mathrm{R}=$ Receiver load resistance

$\mathrm{e}=$ Electron charge

The NEP is defined as the amount of signal power spectral density necessary to make the signal-to-noise ratio in an optical receiver equal to unity.

For a sufficiently large local oscillator power $\left(\mathrm{P}_{\mathrm{LO}}\right)$, equation (A.4) reduces to the quantum, or shot noise limited value of $\mathrm{P}_{\mathrm{Q}} / \mathrm{B}$. For a wavelength of $1550 \mathrm{~nm}$ and a detector quantum efficiency of 0.6 , the shot noise limited NEP is $\sim 2 \times 10^{-19} \mathrm{~W} / \mathrm{Hz}$.

In the case of the CLR sensor, shot noise limited performance represents the most sensitive mode of operation. Thus, it is important to utilize an optical receiver that minimizes the thermal noise and to ensure that the LO power is sufficiency to operate in this mode. 
From equation (A.3) and (A.4), and including a term for excess laser noise, the sensor predetection signal-to-noise ratio (SNR) can be expressed as:

$$
S N R_{I F}=\frac{P_{t} \rho L_{t} d^{2}}{8(N E P)_{H e t} B_{I F} N_{E} R^{2}}
$$

where $N_{E}$ is the laser excess noise factor. This term results from the fact that diode lasers are not ideal "noiseless" sources, but have noise in excess of the quantum noise that is frequency dependent. Typical values for $\mathrm{N}_{\mathrm{E}}$ at frequencies greater than $1 \mathrm{MHz}$ range from 2 to 10 .

For the basic configuration in Figure $2, \mathrm{~L}_{\mathrm{t}} \simeq 0.2$. However, for a more optimal system, as in recent CRC implementations, an optical circulator replaces the $3 \mathrm{~dB}$ coupler and achieves a transmission loss factor of $\mathrm{L}_{\mathrm{t}}=0.8$. For the ITER RMS configuration, there will be up to $6 \mathrm{~dB}$ additional losses in a radiation darkened Bragg cell $3 \mathrm{~dB}$ losses from diffraction inefficiency (both round trip) for a resultant transmission loss factor of $L_{4}=0.1$. The value $L_{1}=0.1$ is used in the RMS performance estimate. Using a laser diode source $\left(P_{t}=1 \mathrm{~mW}\right)$ with an excess noise value of $N_{E}=$ 5 , and a $10 \%$ target $(\mathrm{p}=0.1)$, then equation (A.5) reduces to:

$$
S N R_{I F}=1.25 \times 10^{12}\left(\frac{d}{R}\right)^{2} \frac{1}{B_{I F}}
$$

Equivalently,

$$
\left(S / N_{o}\right)_{I F}=1.25 \times 12\left(\frac{d}{R}\right)^{2}
$$

where $\mathrm{S}=$ Signal power

$\mathrm{N}_{\mathrm{o}}=\mathrm{IF}$ noise power $/ \mathrm{Hz}$

The accuracy of a chirp radar range measurement is determined by the well known accuracy of measuring the frequency of a pure sinusoid IF signal over a measurement interval (chirp period) T: 


$$
\sigma_{f}=\left(\frac{3}{2}\right)^{1 / 2} \frac{1}{\pi T^{3 / 2}\left(\frac{S}{N_{o}}\right)}
$$

This $\sigma_{\mathrm{f}}$, in turn, determines the accuracy of the range measurement:

$$
\sigma_{R}=\frac{\sqrt{2}}{2}\left(\frac{C T}{\Delta f}\right) \cdot \sigma_{f}=\frac{\sqrt{3}}{2} \frac{C}{\pi(\Delta f) T^{1 / 2}\left(\frac{S}{N_{o}}\right)^{1 / 2}}
$$

where $\mathrm{S}=$ Signal power

$\mathrm{N}_{\mathrm{o}}=$ Noise power $/ \mathrm{Hz}$

$\Delta \mathrm{f}=$ Radar FM sweep bandwidth

$\mathrm{T}=$ Sweep period (or measurement integration time if multiple sweeps are integrated)

(A $\sqrt{2}$ factor is added to $\sigma_{\mathrm{R}}$ to account for errors in measuring both the range arm and the reference arm. This is conservative, since reference arm SNR is higher.)

As an example, consider the following parameters:

$$
\begin{aligned}
\Delta \mathrm{f} & =10^{11} \mathrm{~Hz} \\
\mathrm{~d} & =10^{-2} \mathrm{M} \\
\mathrm{R} & =10 \mathrm{M}
\end{aligned}
$$

$$
\text { Then } \sigma_{R}=\frac{7.4 \times 10^{-7}}{T^{1 / 2}}
$$

and we have for accuracy as a function of measurement time:

\begin{tabular}{|c|c|}
\hline$T$ & $\sigma_{R}$ \\
\hline $10^{-4} \mathrm{sec}$ & $0.074 \mathrm{~mm}$ \\
\hline $10^{-3} \mathrm{sec}$ & $0.023 \mathrm{~mm}$ \\
\hline $10^{-2} \mathrm{sec}$ & $0.0074 \mathrm{~mm}$ \\
\hline
\end{tabular}


These parameters are all appropriate or very conservative for the ITER RMS. A linear frequency sweep of $100 \mathrm{GHz}$ in $10^{-3}$ sec has been achieved with a new type of laser diode recently acquired by CRC. An aperture of at least $1 \mathrm{~cm}$ will be used for RMS and measurement ranges will be less then 10M. The output power of these new lasers in $10 \mathrm{mw}$ which could be boosted to $100 \mathrm{mw}$ for the RMS for 100x increase in signal power over that used in this calculation. 